UDC 539.23:548.12

\title{
Obtaining and research of new composite materials polymer-graphene
}

\author{
Myrzabekova M.M., Sarkar S., Baigarinova G.A., Guseinov N.R., Ilyin A.M. \\ Al-FarabiKazakh National University, NNLOT, al-Farabi71,050040 Almaty, Kazakhstan
}

\begin{abstract}
This paper presents the results of computer simulation and calculations of the energy characteristics of several possible stable configurations, providing rigid connection of the polymer matrix with graphene structures in composite materials. The measurements of mechanical and electrical properties of composite sample based on several types of polymers (polyethylene, polyvinyl alcohol, polydimethylsiloxane and epoxy resin) with fillers of graphene and its derivative nanostructures were performed. To study of samples' structure and properties such methods of analysis as Raman spectroscopy, optical microscopy and microhardness testing (Knoop method) were chosen. The results of theoretical calculations of "polymer-graphene structures" systems are in good agreement with preliminary experiments and hardness and strength measurements. Good interconnection of carbon nanostructures with a polymer matrix provides an effective load transfer from the polymer material and the carbon nanostructure, ultimately leads to an increase of the strength characteristics of the composite. It is shown that the addition of graphene and its derivative nanostructures - graphite and graphene oxide - as reinforcing material in a polymer matrix significantly increases the mechanical properties and reduces the electric resistance of the resulting composite.
\end{abstract}

Keywords: graphene, graphene oxide, polymer composites, mechanical properties.

PACS number(s): 78.67.Sc, 81.05.Lg

\section{Introduction}

Carbon nanostructures, in particular graphene and its derivatives have recently been considered as very promising materials for new technologies. To a large extent it relates to the creation of composites based on various types of matrices, for instance polymers or metals. This is due to the fact that graphene and its related structures, having a very favorable combination of physical and mechanical properties of low density are able to give composites completely new physical and mechanical properties. Several recent studies [1-6] have already shown that their use as filler in various matrix materials can significantly change such properties as mechanical strength, stiffness, electrical conductivity, magnetic properties etc. At the same time, in our opinion energy and structural peculiarities of the composite formation of polymer - graphene type are studied insufficiently, which is extremely important for a deeper understanding of the physical mechanisms of the properties modification of matrix - graphene filler systems [7-10]. This piece of work presents the results of theoretical and experimental studies of the physical and mechanical properties of composite materials

\footnotetext{
* Corresponding author: e-mail: ilyinar@mail.ru
}

based on different types of polymers with fillers of graphene and related structures. Computer models of several possible stable configurations for various polymer matrixes filled with fragments of graphene were constructed and quantum mechanical calculations (density functional theory) of their energy and structural characteristics were conducted. Similar calculations for graphene structures with defects predict the possibility of a significant improvement in adhesion to polymers in comparison with the ideal structures. In regard to the experimental part, it shows the results of measurements of mechanical properties and electrical conductivity of polymer composites.

\section{Computer simulations and calculations}

In this part of the article, computer models of the most probable types of possible configurations of graphene and few-layer graphene and polymer molecules are observed. Primarily edges of graphene nanostructures were considered as probable connection zones of the polymer molecules to graphenenanofragments, assuming the existence of a large number of dangling bonds right there. Figure 1 shows possible configurations in which fragments of polyethylene (PE) molecules can be connected with «zig-zag» graphene edge (perpendicular to the edge). 


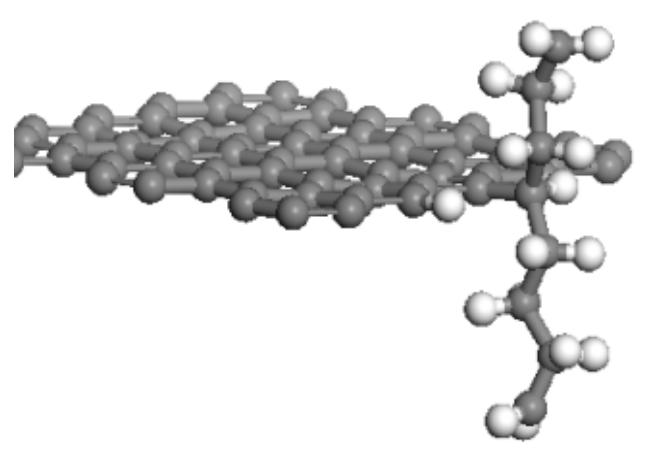

a)

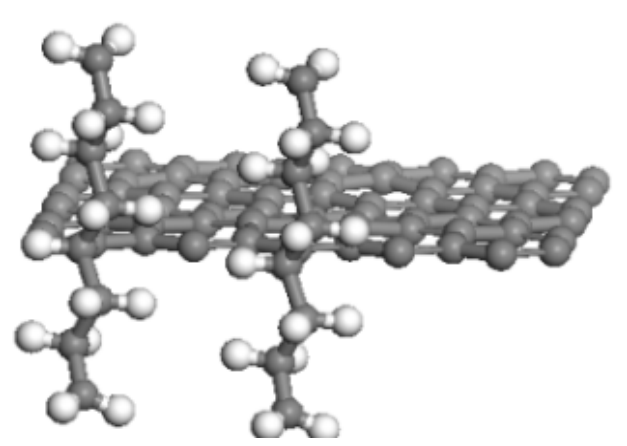

b)

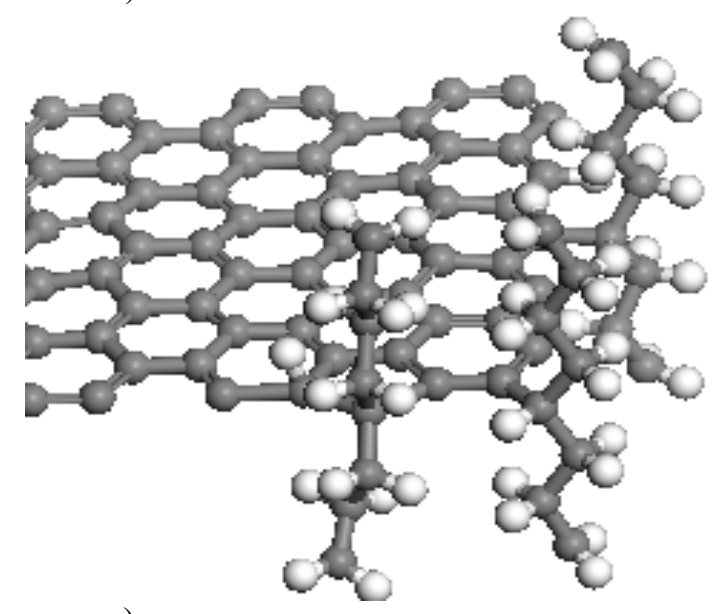

c)

Figure 1 - Configurations of PE molecule - «zig-zag» edge of graphene: a) a single fragment of PE molecule at the edge. The binding energy $\mathrm{Eb}=5,5 \mathrm{eV}$; b) a pair of PE fragments in a parallel arrangement with binding energy $\mathrm{Eb}=5.3 \mathrm{eV}$; c) fragments of molecules $\mathrm{PE}$ attached to the «zig-zag» and «arm-chair».

Studies of the energy properties of such structures have shown that under certain conditions, the formation of covalent bonds between fragments of graphene and molecules of PE are quite possible. To realize this, contact zones between the molecules of $\mathrm{PE}$ and graphene fragments were created (Fig.1). Calculations made by DFT method showed that at the first stage in the contact zone transition of one or two hydrogen atoms out of PE molecule to the boundary carbon atoms in the structure of graphene happens; resulting in dangling bonds appeared on the polymer molecule. After that $\mathrm{C}-\mathrm{C}$ bond is formed between the PE molecule and the boundary graphene atoms. Figure 1 also shows the energetically stable configurations with a compact arrangement of several PE molecules.

Figure 2 illustrates an occasion where a fragment of PE molecule is located parallel to the «zig-zag» edge. It should be noted a distinct change in the spatial arrangement of the hydrogen atoms on the PE molecule and its deformation. Total binding energy of the PE fragment with "zig-zag" edge of graphene for a given geometry is $\mathrm{E}_{\mathrm{b}}=18 \mathrm{eV}$, about $2.5 \mathrm{eV}$ per each $\mathrm{C}$ atom in the molecular chain.

Calculations have shown that at the location of the molecule parallel to the surface of an ideal graphene the binding energy is mainly determined by the Van der Waals interaction and the formation of strong bonds is possible only at the ends of the graphene fragment. Modeling and calculation of defect configuration (vacancy) in the structure of graphene (Fig 2.) showed that in this case, the formation of covalent bonds between the molecule and the carbon atoms in the defect zone quite possible $\left(\mathrm{E}_{\mathrm{b}}=2.5 \mathrm{eV}\right)$. Figure 3 illustrates the configuration of "closing" of typical molecular "loop" in the PE matrix by the graphene fragment.

The calculations confirm that the configuration shown in Figure 3, with the loop closed by the graphene fragment possesses a higher mechanical rigidity of the structure. Figure 4 shows some results of computer modeling of the polydimethylsiloxane (PDMS) - graphene system.

Figure 4 illustrates that in contrast to the $\mathrm{PE}$ molecules, there are two different possible ways of connecting PDMS molecule to graphene for PDMS. Figure $4 \mathrm{a}$ shows the possibility of type $\mathrm{C}-\mathrm{C}$ bond formation between the carbon atoms of the graphene structure and of the polymer molecule, while Figure $4 \mathrm{~b}$ shows a variant of the compound via bond $\mathrm{C}-\mathrm{O}$, which is formed between $\mathrm{C}$ atom from the structure of graphene and the oxygen atom of the polymer molecule. Binding energy for the second configuration is less on $2.1 \mathrm{eV}$. 


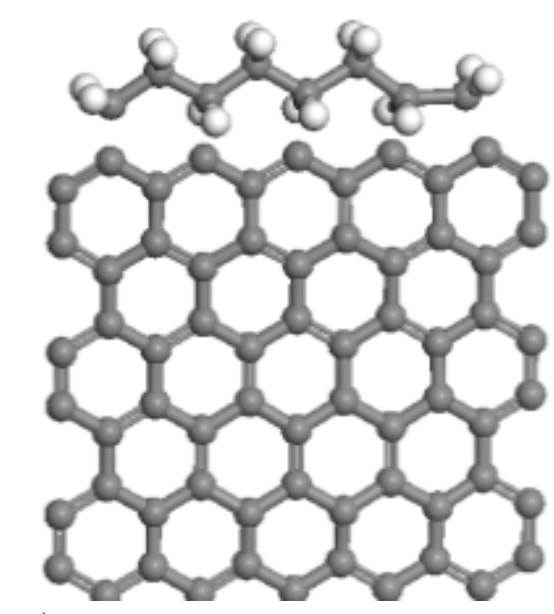

a)

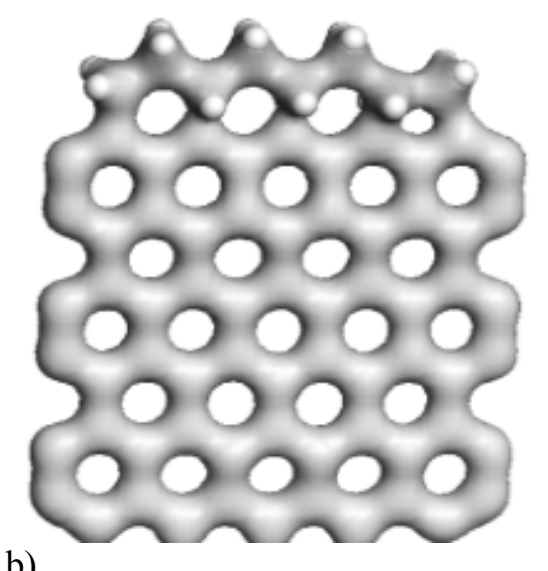

b)

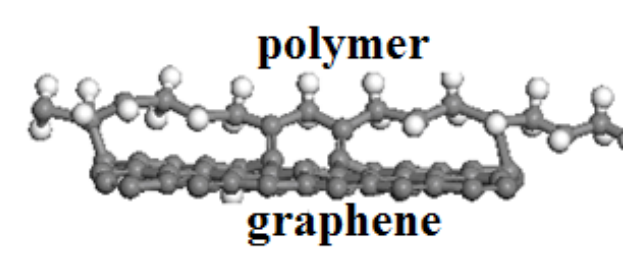

c)

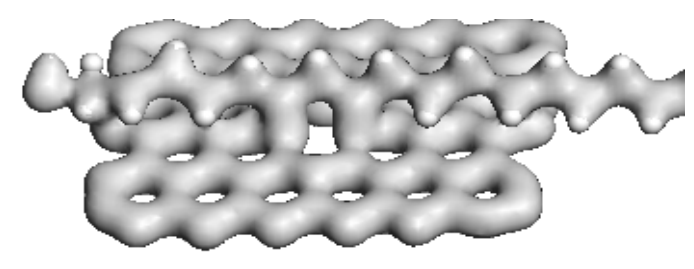

d)

Figure 2 - a) a molecule along «zig-zag»: atomic structure; b) the distribution of electron charge; c) the molecule on the surface of graphene with a vacancy (top); d) the distribution of electron charge (0.7 el / $\AA 3)$.

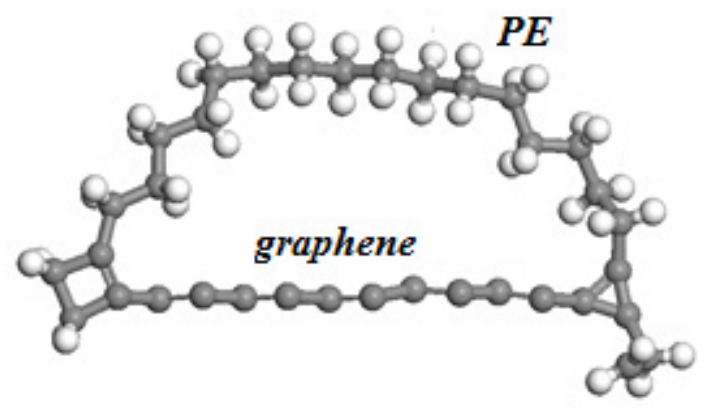

a)

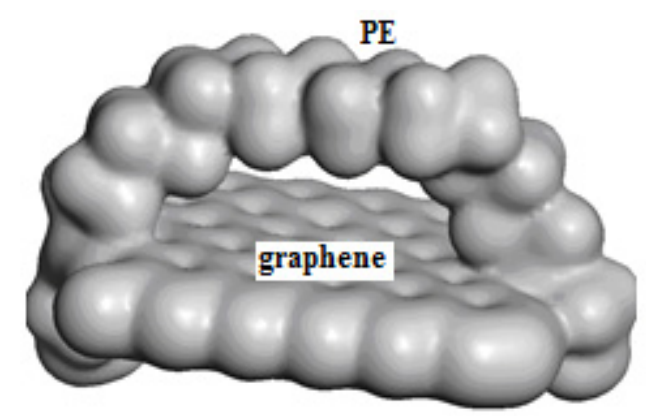

b)

Figure $3-$ a) The atomic structure loop configuration of the molecule PE; b) the distribution of electron charge, density at $\rho=0.7 \mathrm{el} / \AA^{3}$. The binding energy of fragment of the molecule PE graphene $8.5 \mathrm{eV}$.

\section{Experiments}

For creation of composites were used several types of polymer matrix - PE, polyvinyl alcohol (PVA), epoxy resin. Experimental sample of PE was formed as "sandwich" of two thin films of thickness 3 microns, between which graphene fragments were distributed. From the micrographs, shown in Figure 5a it is visible that the distribution of graphene fragments with the density $6 * 10^{6}$ particles $/ \mathrm{cm}^{2}$ was sufficiently uniform.

Further, the PVA is used as the polymer, which is a synthetic, water-soluble, thermoplastic polymer being excellent emulsifying, adhesion and filmforming polymer. It has high tensile strength and flexibility. PVA is stable against oils, greases and organic solvents. Considering indicated qualities PVA was selected for use in experiments as a matrix.

In addition, new composite graphene - PVA has been received in progress. Graphene was obtained by method of chemical vapor-phase deposition on a copper substrate, and then transferred to film. As a result, the composite PVA - graphene - PVA (Fig. 5c) 
was obtained having electrical conductivity (surface resistance is reduced from $10^{12}$ to $25 \mathrm{MOhm} * \mathrm{~cm}$ ), transparency, and high mechanical properties. Figure 6 shows the Raman spectra of graphite, graphene and oxide with $G$ and 2D peaks specific for them.

The next step was to obtain composite materials filled with graphene oxide. The preparation of such composites was conducted by inlet of a certain amount of graphene oxide to the PVA solution. 7a figure shows typical micrographs of such composites. Figure $7 \mathrm{~b}$ illustrates an appearance of a composite based on $\mathrm{E}$ -300 epoxy resin with graphite flakes with an average size of $8 \times 8 \mu \mathrm{m}$.

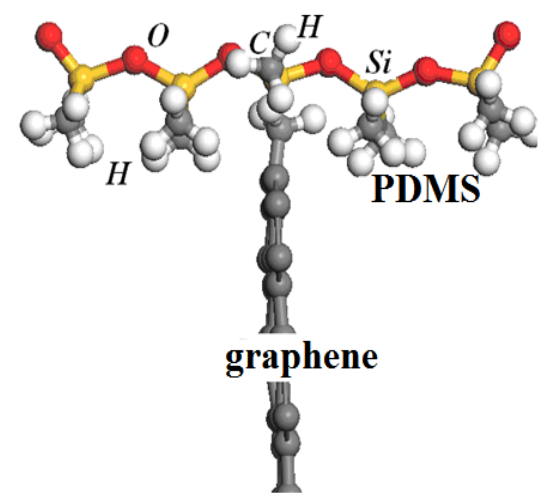

a)

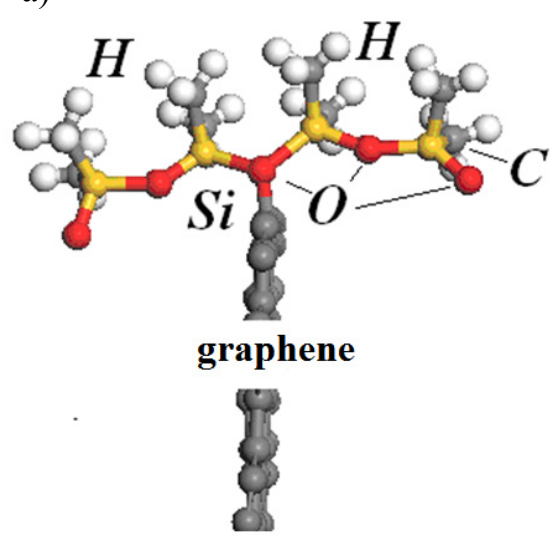

b)

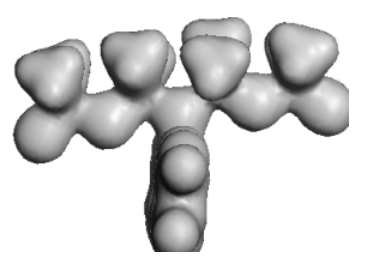

c)

Figure 4 - The model of configuration compounds molecule of PDMS with graphene: a) the bond of type $\mathrm{C}-\mathrm{C}$; b) the bond of type $\mathrm{C}-\mathrm{O} ; \mathrm{c}$ ) the distribution of electron charge, density at $\rho=0.7 \mathrm{el} / \AA \AA 3$.

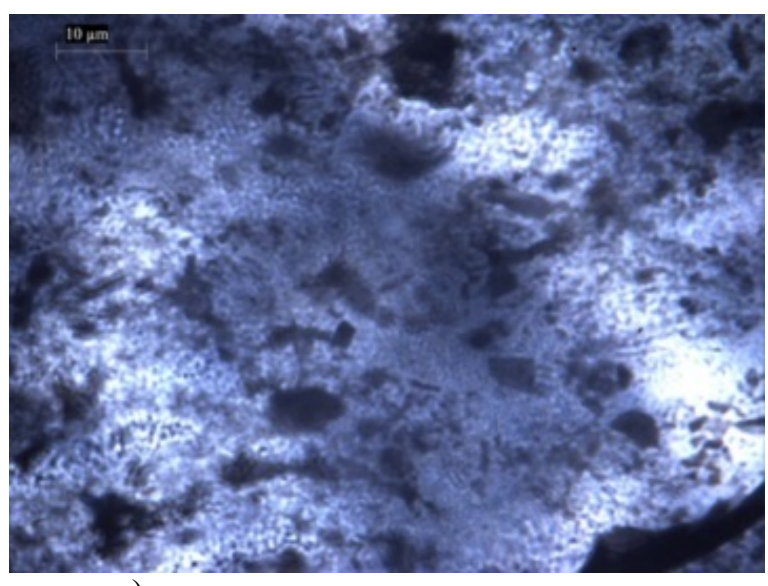

a)

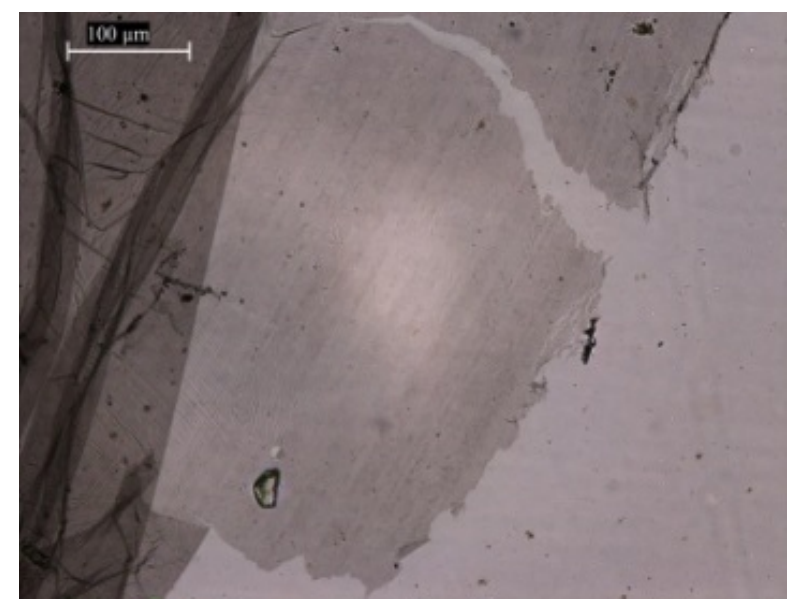

b)

Figure 5 - Optical micrographs of films: a) the composite graphene-polyethylene; b) graphite dust and PVA (reflection).

\section{Discussion of results}

The above presented results of computer modeling and quantum- mechanical calculations of polymer - graphene systems are well correspond with the preliminary experiments data conducted on several types of common industrial polymers. It isshown that the use of graphene and few-layer graphene structures as filler for various matrix materials, allows improving significantly such material properties as mechanical strength, stiffness, conductivity. Figure 8 shows the Knoop (HK) hardness values of composites depending on graphene oxide concentration. 


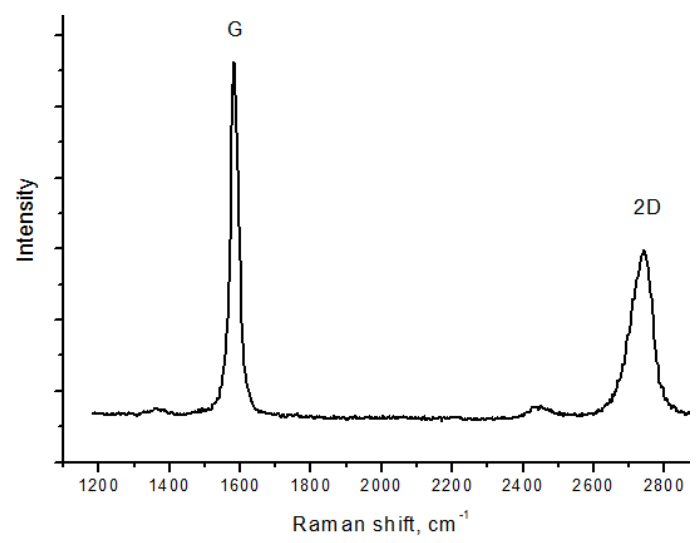

a)

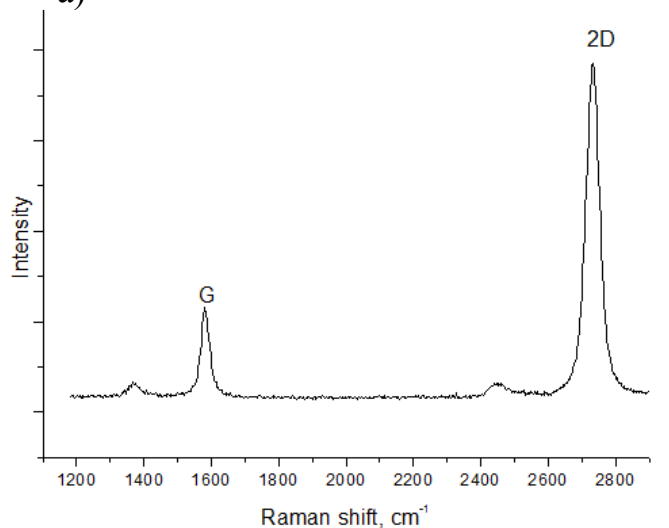

b)

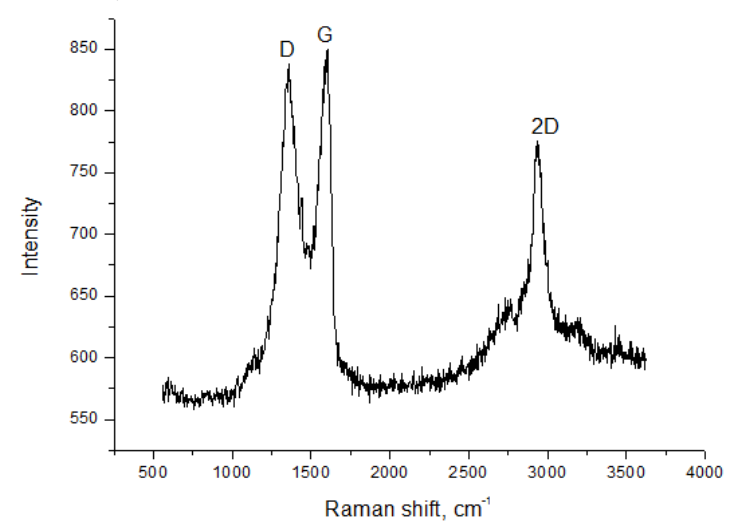

c)

Figure 6 - The Raman spectra with characteristic peaks G and 2D: a) graphite;

b) 8rapheme; c) 8rapheme oxide.

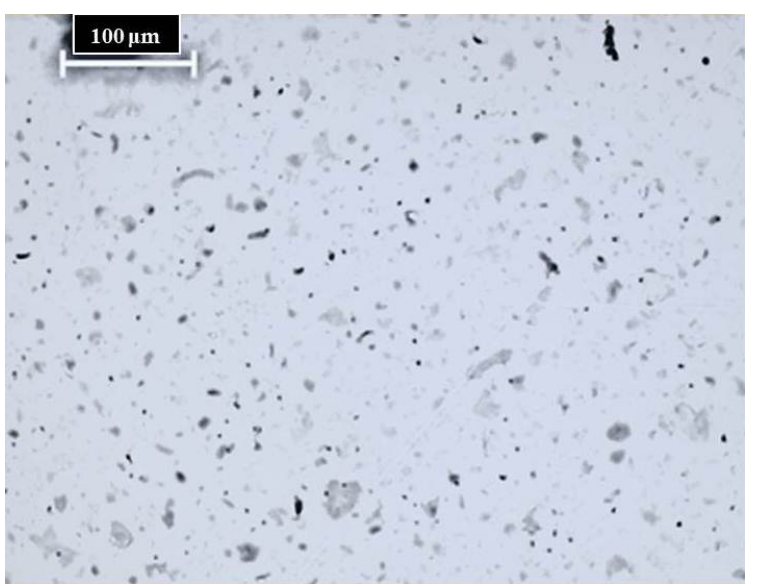

a)

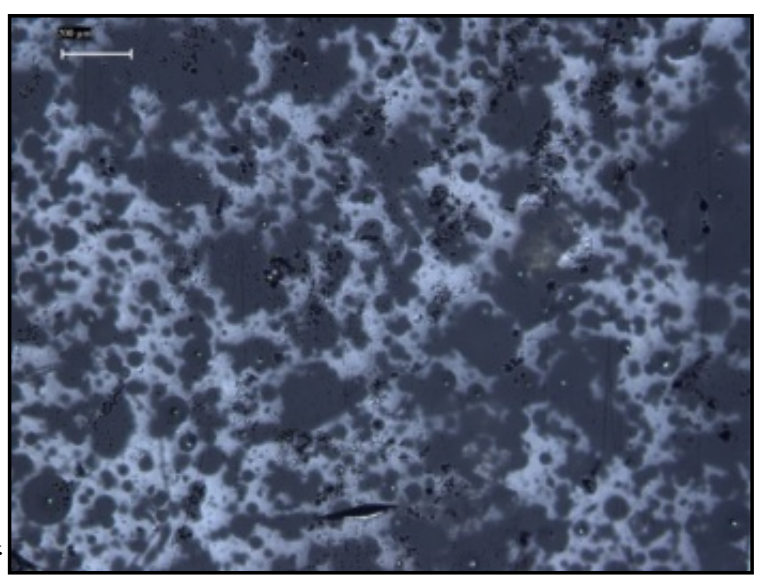

b)

Figure 7 - Optical micrographs of composite films: a) PVA-graphene oxide;

b) epoxy resin - graphite flakes.

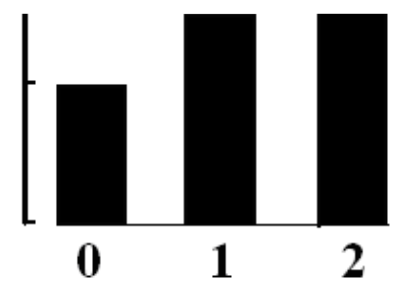

Figure 8 - The histogram of the microhardness values of composite materials based on polymer PVA withgraphene fillers: 0 - original polymer, 1 polymer + graphene oxide $\mathrm{n}=4 * 10^{8} \mathrm{~cm}^{-3}, 2$ polymer + graphene oxide $\mathrm{n}=8 * 10^{8} \mathrm{~cm}^{-3}$ 


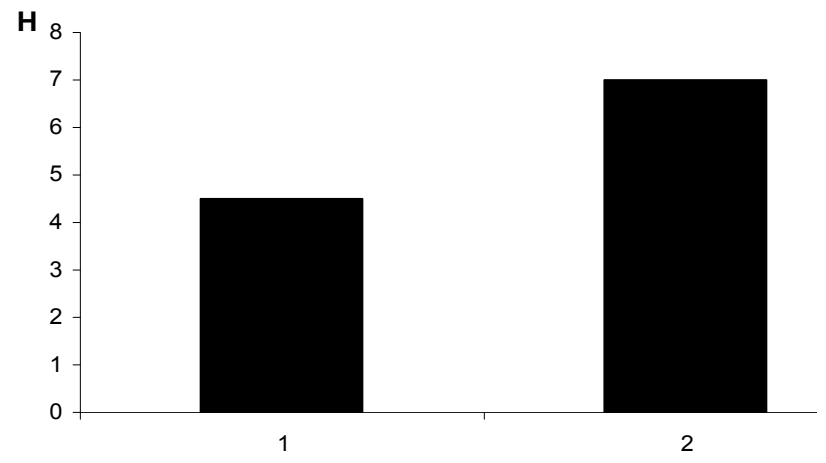

Figure 9 - The histogram of the microhardness values of composite materials based on epoxy resin with graphite flakes: 1 - initial polymer, 2 polymer + graphite

Based on the results of measurements of the microhardness of samples, the addition of graphene structures has significantly increased hardness. Furthermore, the addition of graphene structures also increases the strength characteristics as when tested with high loads the cracks formed on the PVA film and at the adding of few-layer graphene, no cracking is observed at high loads. When you add even more graphene oxide the concentration of graphene structures increases, therefore the hardness values also increases. Figure 9 shows the Knoop (HK) hardness values of epoxy resingraphite composites.

\section{Conclusions}

Measurements of the mechanical properties obtained in the experiments of samples of various polymer composites with graphene fillers are in good agreement with data of recent publications by other authors. Observed in various polymer materials similar changes in physical and mechanical properties prove display of general physical mechanisms operating in the polymer matrix system - graphene structure. To better understand the physical basis of such interaction processes computer modeling was used, several possible configurations were built and quantum mechanical evaluation of the energetic and structural properties of the polymer systems -"fewlayer" graphene was done.

\section{References}

[1] Yang Y., Rigdon W., Huang X., Li X. Enhancing graphene reinforcing potential in composites by hydrogen passivation induced dispersion // Sci. Reports. - 2013. - Vol. 3. - P. 2086.

[2] Stankovich S. et al.Graphene-based composite materials // Nature. - 2006. - Vol. 442. - P. 282-286.

[3] Fang M., Wang K.G., Lu H.B. et al. Covalent polymer functionalization of graphenenanosheets and mechanical properties of composites // J. Mater. Chem. 2009. - Vol. 19. - P. 7098-7105.

[4] Shahil K.M., Balandin A.A. Graphene-multilayer graphenenanocomposites as highly efficient thermal interface materials // Nano Lett. - 2012. - Vol. 12. - P. 861-867.

[5] Chatterjii I.S. et al. Crystalline and tensile properties of carbon nanotubes and graphene reinforced polyamid 12 fibers // Chem.Phys. Lett. - 2013. - Vol. 557. - P. 92. [6] Chatterjii I.S. et al. Mechanical reinforcement and thermal conductivity in expanded graphenenanoplatelets reinforced epoxy composites // Chem.Phys.Lett. - 2012. - Vol. 531. - P. 6.
[7] Ilyin A.M. Computer Simulation of Carbon- and Graphene-Metal Nanostructures: in book series "Computer Science, Technology and Applications"; ed. by Nemanjik B. and Svetozar N. - NY: "Nova_Sci. Publish.”, 2013. - P. 145-160.

[8] Ilyin A.M. Computer Simulation of Radiation Defects in Graphene and Relative Structures: in book "Graphene Simulation"; ed. by Gong J. R. - Croatia: InTech, 2011, - P. 39-52.

[9] Ilyin A.M., Guseinov N.R., Nemkaeva R.R., Tsyganov I.A., Asanova S.B., Kudryashov V.V. BridgeLike Radiation Defects in Few-Layer Graphene // Phys. Res. B. / Nuclear Instruments \&Methods - 2013. - Vol. 315. - P. 192-196.

[10] Ilyin A.M. and Beall G.W. Computer Simulation of Graphene-Metal Composite Induced by Radiation. NanoTech // Composite Materials: Book of proceedings - Boston, USA, June 13-16, 2011. - Vol. 1. - Ch. 5. - P. 574-576. 